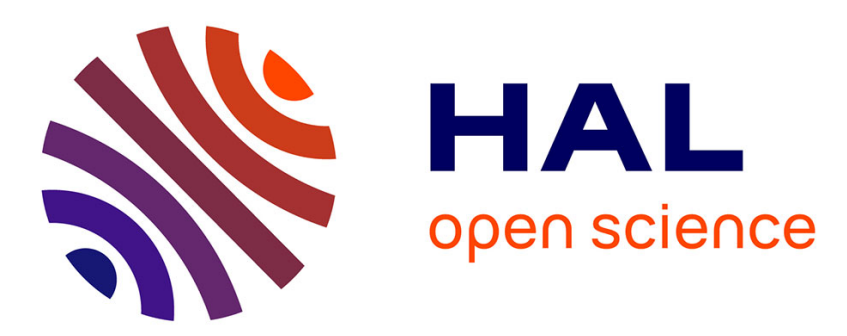

\title{
Observer based multivariable control of a catalytic reverse flow reactor: comparison between $L Q R$ and MPC approaches
}

\author{
David Edouard, Pascal Dufour, Hassan Hammouri
}

\section{To cite this version:}

David Edouard, Pascal Dufour, Hassan Hammouri. Observer based multivariable control of a catalytic reverse flow reactor: comparison between LQR and MPC approaches. Computers \& Chemical Engineering, 2005, 29 (4), pp.851-865. 10.1016/j.compchemeng.2004.09.018 . hal-00091715v2

\author{
HAL Id: hal-00091715 \\ https://hal.science/hal-00091715v2
}

Submitted on 21 Jan 2009

HAL is a multi-disciplinary open access archive for the deposit and dissemination of scientific research documents, whether they are published or not. The documents may come from teaching and research institutions in France or abroad, or from public or private research centers.
L'archive ouverte pluridisciplinaire HAL, est destinée au dépôt et à la diffusion de documents scientifiques de niveau recherche, publiés ou non, émanant des établissements d'enseignement et de recherche français ou étrangers, des laboratoires publics ou privés. 
This document must be cited according to its final version which is published in a journal as:

D. Edouard ${ }^{1}$, P. Dufour ${ }^{1}$, H. Hammouri ${ }^{1}$, "Observer based multivariable control of a catalytic reverse flow reactor: comparison between LQR and MPC approaches", Computers and Chemical Engineering, ISSN: 0098-1354 29(4), pp. 851-865, 2005. This final version may be found: http://dx.doi.org/10.1016/j.compchemeng.2004.09.018

All open archive documents of Pascal Dufour are available at: http://hal.archives-ouvertes.fr/DUFOUR-PASCAL-C-3926-2008

The professional web page (Fr/En) of Pascal Dufour is: http://www.lagep.univ-lyon1.fr/signatures/dufour.pascal

1

Université de Lyon, Lyon, F-69003, France; Université Lyon 1;

CNRS UMR 5007 LAGEP (Laboratoire d'Automatique et de GEnie des Procédés),

43 bd du 11 novembre, 69100 Villeurbanne, France

Tel +33 (0) 472431845 - Fax +33 (0) 472431699

http://www-lagep.univ-lyon1.fr/ http://www.univ-lyon1.fr http://www.cnrs.fr 


\title{
Observer based multivariable control of a
}

\section{catalytic reverse flow reactor: comparison}

\section{between LQR and MPC approaches}

\author{
Edouard D. ${ }^{\mathrm{a}}$, Dufour P. ${ }^{\mathrm{b}, 1}$, Hammouri H. ${ }^{\mathrm{b}}$ \\ ${ }^{\mathrm{a}} L G P C, C P E$ Lyon, UMR CNRS 2214 \\ 43 Bd du 11 Novembre 1918 \\ 69622 Villeurbanne Cedex France. \\ ${ }^{\mathrm{b}}$ LAGEP, Université Claude Bernard Lyon 1 UMR CNRS 5007 \\ 43 Bd du 11 Novembre 1918 \\ 69622 Villeurbanne Cedex France.
}

\begin{abstract}
This paper is intended to show the comparison of two closed-loop controllers performances for a catalytic reverse flow reactor (RFR): a linear quadratic regulator (LQR) and a model predictive control (MPC) strategy that both use a high-gain observer. The aim of the RFR is to reduce, by catalytic reaction, the amount of volatile organic compounds (VOCs) released in the atmosphere. The particularity
\end{abstract}


of this process is that the gas flow inside the reactor is periodically reversed in order to trap the heat released during the reaction inside the process. Very few papers are dealing with the control of the RFR. The new multivariable optimal control issue tackled here is to confine the hot spot temperature within two temperature limits, in order to ensure complete conversion of the pollutant and to prevent catalyst overheating, while optimizing the process yield and the consumption of electrical power. Both control laws aim to optimize the tuning of the dilution and the internal electric heating. The model considered here for control of the RFR is obtained from a countercurrent pseudo-homogeneous partial differential equation (PDE) model. The feed concentration (acting as an important input disturbance) and the temperature profile in the RFR are estimated on-line using a high-gain observer based on three temperatures measurements. In order to maintain the safe use of the reactor, the estimate state is injected in the LQR whereas the MPC is based on the estimated input disturbance. Simulation results allow comparing performances of the LQR and the MPC.

Key words:

Multivariable control of a catalytic reverse flow reactor, control of a nonlinear distributed parameter system, LQR, MPC, observer design.

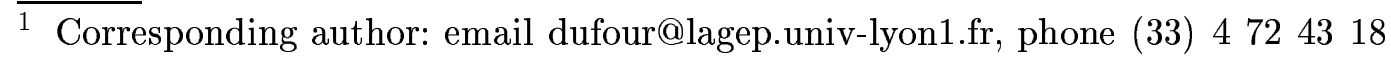
78, fax (33) 472431699 


\section{Introduction}

This paper deals with the multivariable optimal control of a catalytic reverse flow reactor, which topic has not been often be tackled until now. The RFR aims to destruct VOCs. Even if the definition of VOCs is blurred, it includes noxious products whose chemical reactivity is likely to influence atmospheric pollution. For this reason, they are the source of a lot of environmental problems including: acid rains, woods wasting, greenhouse effect and health hazards. Problems of environmental pollution due to the industrial production are therefore receiving increased attention and due to the public regulations, VOCs discharge in the atmosphere becomes strictly limited. Therefore, the VOCs emission reduction represents a priority, especially since the problem is connected with a large field of activities from large-scale factories to small and medium-sized firms like dry cleaners.

In a previous work, the single input single output (SISO) control of the RFR was treated [12]. The aim was to control the RFR such that the outlet gas concentration released in the atmosphere was maintained below a maximum level fixed by public regulations. This control strategy was based on a parabolic PDE model, an internal model control (IMC) structure and a MPC framework. According to various regimes, it was shown that the proposed controller was able to tune correctly the control action, i.e. the heating power at the core of the reactor. In the meantime, for a particular regime, the temperature inside the reactor was exceeding a threshold temperature that reflects the deterioration of the catalytic elements. Moreover, the input disturbance was assumed to be relatively constant and measured, whereas unmeasured and large stochastic variations need to be accounted for in reality. The aim of this 
paper is to provide a multivariable control framework to solve these problems. This requires modifying the PDE model and the control problem to account for a new manipulated variable: the cooling action.

The RFR is modelled by a nonlinear PDE system characterized by complex nonlinearities in the spatial domain. Even with only one spatial dimension, control of PDE systems is not often treated, especially in the nonlinear case. Explicitly, transport reaction phenomena with significant diffusive and convective phenomena are typically characterized by severe nonlinearities and spatial variations, and are naturally described by partial differential equations. Examples of such processes include tubular reactors, packed bed reactors, absorption columns, drying or curing processes. In control theory, due to the complexity of the problem, relatively few studies are devoted to the control of processes explicitly characterized by a PDE model. Even if various methods are proposed to control such distributed parameter systems, there is no general framework yet. In order to implement, with a computer, a low order model based controller, the original PDE model is usually simplified into an ordinary differential equation (ODE) model. Such a finite dimensional approximation is based on the finite differences method, the finite volume method, the orthogonal collocation method or the Galerkin's method. Other works utilized properties of the initial PDE system before finite dimension controller synthesis: Recently, Christofides developed order reduction by partitioning the eigen spectrum of the operator of the PDE system $[8,17]$ and methods based on approximate inertial manifold for spatial discretization of the PDE $[7,2]$. Other works for controller synthesis of nonlinear PDE systems are based on symmetry groups, infinitesimal generators and invariant conditions [32,21]. Concerning $[22,40]$, finite dimensional controllers are obtained through model reduction based on various methods: singular value decomposition, Karhunen- 
Loéve expansion or eigenfunction method. With this method, an interesting framework is provided with proof of closed-loop stability for the QDMC of a PDE system [41]. In [3], stability conditions for closed-loop control of linear PDE with finite dimensional controller are given in time domain and frequency domain through semigroup analysis. In [38], based on semigroup theory, proofs were given for the closed-loop stability of PI control for a linear PDE system. This paper is not intended to propose a new PDE model based control framework but rather to show how advanced control strategies may be used for the control of the RFR. Indeed, few works are dealing with the control of such process described by a nonlinear PDE model. In this paper, two modelbased control strategies for the constrained optimal control of the RFR are compared: LQR and MPC which both use a high-gain observer. The LQR is used here in a classical framework [25]. MPC was developed for ordinary differential equation models and is well dedicated to solve a constrained problem $[1,29,27,36,34]$. MPC was applied for a few PDE systems in $[15,33,37,23]$, where accurate high order dimension models are accounted for. Very recently [41], singular value decomposition and Karhunen-Loéve expansion was used in a QDMC framework. In this paper, we use a MPC strategy $[12,13]$ that aims to reduce, during the sampling period, the on-line calculation time due to the PDE model based optimization task resolution. This approach is based on a strategy combining the IMC structure and a two-phase approach to account for an approximated model into the controller. This approximated model combines the nonlinear PDE model solved off-line and a time-varying linearized PDE model solved on-line. Such MPC approach combined with IMC, even if a low order model is used to approximate the model, allows reaching the required closed-loop performances [14].

The paper is structured as follows: in section 2, the RFR, the new PDE model 
and the discretized model are presented. Section 3 aims to remind the observer previously designed. Section 4 deals with the LQR and MPC strategies used here. Finally, simulation results given in section 5 allow comparing the performances of the LQR and the MPC.

\section{Process and modelling}

\subsection{Process description}

A medium-scale RFR for VOC combustion, as schematically shown in Figure 1 (see [35] for instance) has been considered in this work. Cordierite monoliths (corning) of square cross sections with channels of $1 \times 1 \mathrm{~mm}$ are packed in the reactor. Monolith in the core region is catalytically active and is inert in both end sections. A blower located downstream of the RFR keeps aspiration at a constant flow rate. Liquid pollutant (xylene), is injected into ambient air through a capillary tube and is then vaporized before entering the catalytic layer. An electric heater is installed in the core region to increase the temperature, while fresh air dilution (which results in a small feeding rate in the upstream) is employed to reduce the temperature. Fast flow reversal (the period is $16 s$ ) is needed to keep the highest temperature moving in the catalytic region $[30,31]$. The reactor is encapsulated in a rectangular box and is thermally well insulated. Therefore the packed layer is adiabatic, except in the core region where heat loss is inevitable due to both the installation for air dilution and the high temperature in this region. Moreover, the core is not

airtight (even if it is closed) and, as a result of leakage, a small net amount of fresh air is aspirated into the core: $5 \mathrm{~m}^{3} / \mathrm{hr}$ when the flow rate is $100 \mathrm{~m}^{3} / \mathrm{hr}$. 
Three thermocouples are installed in the reactor and will be used to estimate the temperature profile and the inlet pollutant concentration. The reverse flow

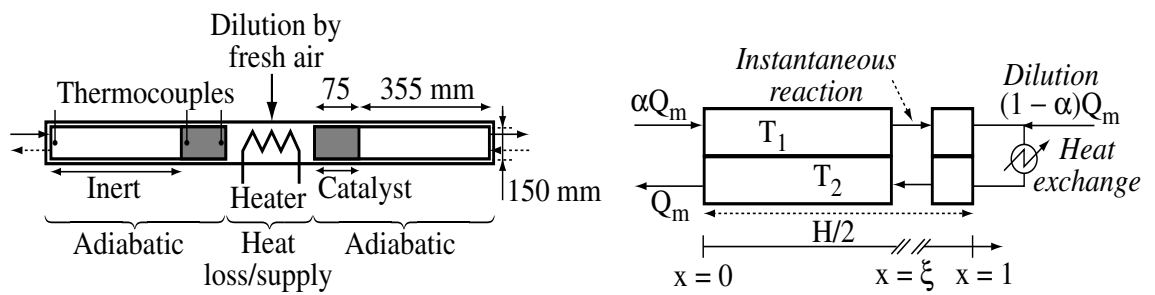

Fig. 1. Left: main geometrical characteristics of the RFR. Right: the countercurrent model.

reactor used here allows high temperatures in catalyst bed whereas the inlet and outlet gas stream temperatures are close to ambient temperature. Indeed, through periodic flow reversal, the heat released by the reaction is first trapped in the packing and is then used to heat up the feed when the flow direction is reversed. Because of the high efficiency of heat exchange between gas and solid phases, autothermal operation is possible even for a feed with a low adiabatic temperature rise (below $15 K$ ). Moreover, owing to the large heat capacity of the packing, the high temperature plateau established in the packed bed is poorly sensitive to abrupt changes in the inlet concentration. These features make therefore RFR highly competitive for VOCs combustion.

\subsection{Countercurrent pseudo-homogeneous model}

Taking advantage of the high frequency of flow reversal, this reactor can be approximated by the countercurrent reactor model [31], as illustrated in Figure 1. This countercurrent reactor model is a heterogeneous model described by a set of three nonlinear PDEs and one algebraic equation. In order to homogenize and simplify this model, the following model has been introduced by Edouard 
et al [16]. It is based on the method described in [4] and it assumes that the kinetic reaction can be neglected under strong mass transfer limitation. It allows obtaining the following pseudo-homogeneous model described by (1)(5): it features one PDE, two algebraic equations to account for mass transfer limitation, and a periodic frequency correction. Normalizing some variables, we obtain:

$$
\left\{\begin{array}{l}
\left(\frac{1}{P_{a x_{\theta}}}+\frac{1+\alpha^{2}}{2 P_{\theta}}\right) \frac{\partial^{2} T_{s}(x, t)}{\partial x^{2}}+\frac{1-\alpha}{2} \frac{\partial T_{s}(x, t)}{\partial x}+P_{\theta} \Delta T_{a d}(t) \psi(x, t)=\tau \frac{\partial T_{s}(x, t)}{\partial t} \\
\alpha \frac{\partial \omega_{1}(x, t)}{\partial x}+P_{\theta} \omega_{1}(x, t)=0, \quad-\frac{\partial \omega_{2}(x, t)}{\partial x}+P_{\theta} \omega_{2}(x, t)=0
\end{array}\right.
$$

with:

$$
\left\{\begin{array}{l}
P_{\theta}=\frac{h a_{c} H}{2 \rho_{0} u_{v 0} c_{p m g}}\left(1-\frac{\theta}{2 \tau}\right), \frac{1}{P_{a x_{\theta}}}=\frac{2 \lambda_{a x}^{s}}{\left(H \rho_{0} u_{v 0} c_{p m g}\right)\left(1-\frac{\theta}{2 \tau}\right)}, \\
\tau=\frac{(1-\varepsilon) \rho_{s} c_{p s} H}{2 \rho_{0} u_{v 0} c_{p m g}}, \Delta T_{a d}(t)=\frac{\Delta H}{M c_{p m g}} \omega_{10}(t), \quad \psi(x, t)=\varphi(x) \frac{\omega_{1}(x, t)+\omega_{2}(x, t)}{2 \omega_{10}(t)}, \\
x=\frac{z}{H / 2}
\end{array}\right.
$$

where $x$ is the normalized space variable, $\varphi(x)$ accounts for the type of monoliths: $\varphi(x)=0$ in the inert monoliths $\left(x<\xi_{\theta}\right)$ and $\varphi(x)=1$ in the catalytic monoliths $\left(x \geq \xi_{\theta}\right)$. The boundary conditions are:

at $x=0$ :

$$
\left\{\begin{array}{l}
\omega_{1}(x, t)=\omega_{10}(t) \\
T_{g 1}(x, t)=T_{s}(x, t)-\frac{\alpha}{P_{\theta}} \frac{\partial T_{s}(x, t)}{\partial x}=T_{0}
\end{array}\right.
$$


at $x=1$ :

$$
\left\{\begin{array}{l}
\left(1+N^{\prime}\right)\left(T_{g 2}(x, t)-T_{0}\right)=\alpha\left(T_{g 1}(x, t)-T_{0}\right)+\frac{Q_{j}}{S \rho_{0} u_{v 0} C_{p m g}} \\
T_{g 1}(x, t)=T_{s}(x, t)-\frac{\alpha}{P_{\theta}} \frac{\partial T_{s}(x, t)}{\partial x} \\
T_{g 2}(x, t)=T_{s}(x, t)+\frac{1}{P_{\theta}} \frac{\partial T_{s}(x, t)}{\partial x} \\
\alpha \omega_{1}(x, t)=\omega_{2}(x, t)
\end{array}\right.
$$

Initial conditions are at $t=0$ :

$$
T_{g 1}(x, t)=T_{g 2}(x, t)=T_{s}(x, t)=T_{0}
$$

In the above equations, $T_{0}$ is the ambient temperature and the feed temperature, $T_{s}$ is the solid temperature and $T_{g 1}$ and $T_{g 2}$ are upstream and downstream gas temperatures respectively. Heat loss, in terms of transfer units $N^{\prime}$, dilution rate $(1-\alpha)$ (percentage of fresh air in downstream flow), and heating power $Q_{j}$ (in watts) are accounted for in the boundary condition at $x=1$.

The first term in the left hand side of (1) involves an effective axial heat conductivity given by:

$$
\left\{\begin{array}{l}
\frac{1}{P_{a x_{\theta}}}+\frac{1+\alpha^{2}}{2 P_{\theta}}=\frac{\lambda_{e f f}}{\rho_{0} u_{v 0} c_{p m g} H / 2\left(1-\frac{\theta}{2 \tau}\right)} \\
\lambda_{e f f}=\lambda_{a x}^{s}+\frac{1+\alpha^{2}}{2} \frac{\left(\rho_{0} u_{v 0} c_{p m g}\right)^{2}}{h a_{c}}
\end{array}\right.
$$

When $\alpha=1$ (i.e. there is no dilution), $\lambda_{\text {eff }}$ reduces to the well-known estimate of [39] as used by Nieken et al. [31]. Finally, this model has been shown to experimentally match the process behaviour during open-loop control and identification [16]. 
The discretization techniques used to solve the model [16] is based on the finite difference method. The discretization points are denoted by $x_{i}$. In order to obtain a satisfactory temperature profile, simulation requires 201 discretization points. $\left(T_{s}\left(x_{0}, t\right), \ldots, T_{s}\left(x_{100}, t\right)\right)$ and $\left(T_{s}\left(x_{101}, t\right), \ldots, T_{s}\left(x_{201}, t\right)\right)$ are the respective discretized temperature profile in the inert monolith and the catalytic monolith. The temperature $T_{s}\left(x_{0}, t\right)$ and $T_{s}\left(x_{201}, t\right)$ are given by the boundary conditions (3) and (4). In the sequel, we will use the following notation:

$X^{1}(t)=\left(\begin{array}{c}T_{s}\left(x_{1}, t\right) \\ \vdots \\ T_{s}\left(x_{100}, t\right)\end{array}\right)=\left(\begin{array}{c}X_{1}^{1}(t) \\ \vdots \\ X_{100}^{1}(t)\end{array}\right)$

$X^{2}(t)=T_{s}\left(x_{101}, t\right)$

$X^{3}(t)=\left(\begin{array}{c}T_{s}\left(x_{102}, t\right) \\ \vdots \\ T_{s}\left(x_{200}, t\right)\end{array}\right)=\left(\begin{array}{c}X_{1}^{3}(t) \\ \vdots \\ X_{99}^{3}(t)\end{array}\right)$

With this notation, the candidate discretized system used for the control takes the following form: 


$$
\left\{\begin{array}{l}
\dot{X}^{1}(t)=A^{1}(\alpha(t)) X^{1}(t)+G^{1}(\alpha(t)) X^{2}(t)+d^{1}(\alpha(t)) T_{0}(t) \\
\dot{X}^{2}(t)=A^{2}(\alpha(t)) X^{2}(t)+G_{1}^{2}(\alpha(t)) X_{100}^{1}(t)+G_{2}^{2}(\alpha(t)) X_{1}^{3}(t) \\
+d^{2}(\alpha(t)) \Delta T_{a d}(t) \\
\dot{X}^{3}(t)=A^{3}(\alpha(t)) X^{3}(t)+G^{3}(\alpha(t)) X^{2}(t)+B^{3}(\alpha(t)) Q_{j}(t) \\
+d_{1}^{3}(\alpha(t)) \Delta T_{a d}(t)+d_{2}^{3}(\alpha(t)) T_{0}(t)
\end{array}\right.
$$

$A^{i}, B^{i}, G_{i}^{i}$ and $d_{i}^{i}$ expressions are given in the appendix.

\section{High-gain observer}

In practice, a model-based control or a supervision strategy may require the knowledge of the temperature profile inside the reactor. One way to obtain such unknown state consists in using physical sensors. However, in many cases, due to cost consideration and physical constraints, the number and types of sensors may be very limited. To avoid this problem, one solution is to design an observer. This method combines a priori knowledge about a physical system (nominal model) with experimental data (some on-line measurements) to provide an on-line estimation of the state and/or the model parameters. In our case, the LQR requires the temperature profile and the MPC strategy is based on the inlet concentration. The observer described and experimentally validated by Edouard and al [16] permits to estimate on-line both the inlet concentration and the temperature profile. The pollutant concentration is considered here as an unknown disturbance to estimate. Edouard et al [16] assumes that $\Delta T_{a d}(t)$ can be considered as the response of a second order 
system. This assumption is not a strong one since any physical signal can be approximated by a response of such a second order filter. The model used to design this observer is therefore a combination of the model (7) and the following second order system:

$$
\left\{\begin{array}{l}
\frac{d}{d t} \Delta T_{a d}(t)=\zeta(t) \\
\frac{d}{d t} \zeta(t)=v(t)
\end{array}\right.
$$

where $v(t)$ is an unknown and bounded signal. The observer synthesis derives from the high-gain techniques (see for instance $[5,11,20,18]$ ). Only three temperature measurements are available for the observer design: the temperature at the inlet of the inert monolith $\left(T_{s}\left(x_{1}, t\right)\right)$, the temperature at the inlet of the catalytic monolith $\left(T_{s}\left(x_{101}, t\right)\right)$ and the temperature at the outlet of the catalytic monolith $\left(T_{s}\left(x_{200}, t\right)\right)$.

In the sequel, we use the following notations for the estimate state $X_{e}(t)$ :

$$
\begin{aligned}
& X_{e}(t)=\left(\begin{array}{c}
X_{e}^{1}(t) \\
X_{e}^{2}(t) \\
X_{e}^{3}(t)
\end{array}\right), \text { where } X_{e}^{1}(t)=\left(\begin{array}{c}
X_{e 1}^{1}(t) \\
\vdots \\
X_{e 1}^{3}(t) \\
X_{e 100}^{1}(t)
\end{array}\right) X_{e}^{2}(t)=\left(\begin{array}{c}
X_{e 1}^{2}(t) \\
X_{e 2}^{2}(t) \\
X_{e 3}^{2}(t)
\end{array}\right) \text { and } \\
& X_{e}^{3}(t)=\left(\begin{array}{c}
X_{e} \\
X_{e 99}^{3}(t)
\end{array}\right)
\end{aligned}
$$


are respectively the state estimates of the vector states $X^{1}(t),\left(\begin{array}{c}X^{2}(t) \\ \Delta T_{a d}(t) \\ \zeta(t)\end{array}\right)$ and $X^{3}(t) . \Delta T_{a d}(t)$ and $\zeta(t)$ are given in (8).

The output measurements are denoted by $y_{1}(t)=X_{1}^{1}(t)=T_{s}\left(x_{1}, t\right), y_{2}(t)=$ $X_{1}^{2}(t)=T_{s}\left(x_{101}, t\right)$ and $y_{3}(t)=X_{99}^{3}(t)=T_{s}\left(x_{200}, t\right)$. With these notations, the high-gain observer takes the following structure:

$$
\left\{\begin{array}{l}
\dot{X}_{e}^{1}(t)=A^{1}(\alpha(t)) X_{e}^{1}(t)+G^{1}(\alpha(t)) y^{2}(t) \\
+d^{1}(\alpha(t)) T_{0}(t)+L^{1}(\alpha(t))\left(X_{e 1}^{1}-y_{1}(t)\right) \\
\dot{X}_{e}^{2}(t)=\widetilde{A}^{2}(\alpha(t)) X_{e}^{2}(t)+G_{1}^{2}(\alpha(t)) X_{e 100}^{1}(t)+G_{2}^{2}(\alpha(t)) X_{e 1}^{3}(t) \\
+L^{2}(\alpha(t))\left(X_{e 1}^{2}-y_{2}(t)\right) \\
\dot{X}_{e}^{3}(t)=A^{3}(\alpha(t)) X_{e}^{3}(t)+G^{3}(\alpha(t)) y_{2}(t) \\
+B^{3}(\alpha(t)) Q_{j}(t)+d_{1}^{3}(\alpha(t)) X_{e 2}^{2}(t)+d_{2}^{3}(\alpha(t)) T_{0}(t)+L^{3}(\alpha(t))\left(X_{e 99}^{3}-y_{3}(t)\right)
\end{array}\right.
$$

The matrices $A^{1}, \widetilde{A}^{2}$ and $A^{3}$ and the observer gains $L^{1}, L^{2}$ and $L^{3}$ are given in the appendix. This observer has been experimentally validated [16]: it gives a satisfactory estimation of both the temperature profile and inlet concentration (even when very stochastic variations (see Figure 2) occur). This observer is used here in both control strategies: to estimate the state required by the LQR and to estimate the input disturbance $\Delta T_{a d}(t)$ required in the MPC. 


\section{Model based control strategies of the RFR}

According to the operating conditions, various problematic behaviours can take place during the operation of the RFR:

- When the feed of pollutant concentration is too rich, the release of heat due to the reaction produces thermal overheating that deteriorates the catalysts. The temperature inside the reactor has therefore to be maintained under the maximum temperature specified as $600 K$ [35].

- If the feed of pollutant concentration is too lean, low heat released during the reaction leads to the extinction of the reactor. The temperature inside the reactor has therefore to be maintained over the lowest temperature specified as $450 K[35]$.

- Ideal operation of such reactor is finally an operation without control. This is possible when the feed concentration is such that the reaction temperature falls within an envelope outlined by the two previous boundary temperatures. This ideal case is called autothermal operation and no control action is required.

Except for the autothermal case, the controller to design has to compensate the influence of the input disturbance $\Delta T_{a d}$ while preserving the stability of the system. On the other side, most of the reaction takes place at the inlet of the catalytic monoliths and is instantaneous $[30,31,35]$. Therefore, instead of the full temperature profile, only $T_{s}\left(x_{101}, t\right)$ has to be maintained between $450 \mathrm{~K}$ and $600 K$. Concerning the input disturbance, $\Delta T_{a d}$ varies between $0 K$ and $30 K$ in real industrial use. But in order to prevent any accident, worst cases have to be evaluated. The equivalent concentration $\Delta T_{a d}$ is therefore assumed 
to vary randomly between $0 K$ and $115 K$ (Figure 2 ) and if no control is applied to the RFR, the hot-spot temperature can not be maintained between both temperature limits (450K and $600 K$ ) (Figure 3). This clearly justifies the need for closed-loop control. 


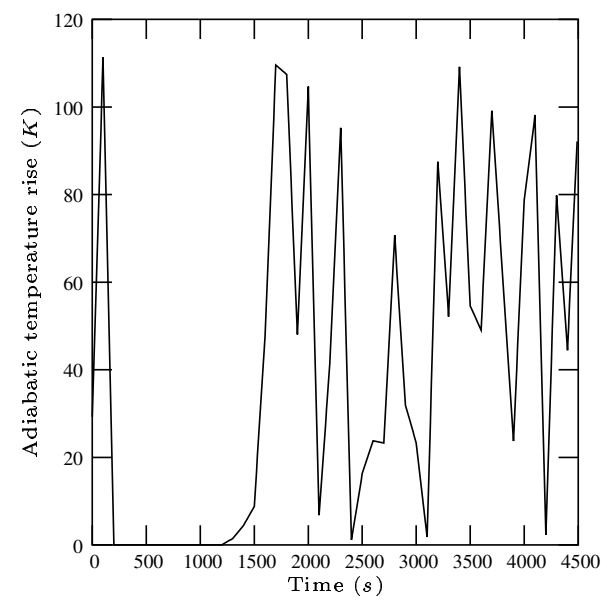

Fig. 2. Input disturbance.

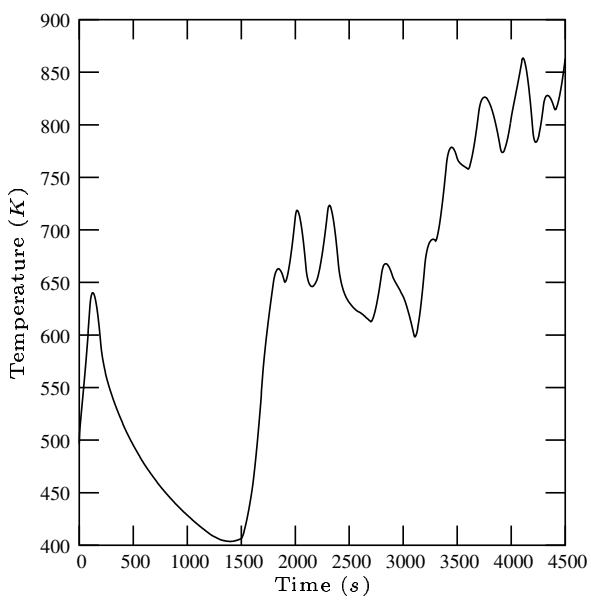

Fig. 3. Hot spot temperature without control. 


\subsection{Overview of RFR control}

Different reactor configurations of RFR have been proposed to provide efficient means of temperature control [31]. For rich feed, one can use cold gas injection, hot gas withdrawing, or heat recovery through internal heat exchangers to suppress temperature run-away; while for lean feed, hot gas supply, or internal heating can be applied to prevent extinction [10]. Nieken and al [30] demonstrated that structured packing with catalytic, less thermal conductive inert part and more thermal conductive outer part is more effective to reduce the maximum temperature than hot gas withdrawal. It is also efficient to prevent early extinction by ensuring an efficient heat exchange between feed and effluent. The first complete study on RFR control has been written by Budman et al. [6]. In this paper, a parametric study of the reactor allows characterizing the operating use of the reactor with respect to two manipulated variables: the coolant flow rate and the cycle time. Moreover, Budman et al. developed two SISO control approaches (coolant flow rate is the manipulated variable) in the case where temperature and concentration at the reactor inlet where assumed to be constant input disturbances. First, a PID controller, based on a local linear model, is given. Secondly, a feed forward controller is given but it is not usable during transient conditions and it is not robust with respect to modelling errors. [9] gives some guidelines for the control of such process accounting for auto thermal and overheating phenomena. Recently, to avoid extinction and overheating, [24] proposed a simple switching control law strategy by on-line tuning of the switching time. In our previous work [12], SISO control strategy was developed to minimize the consumption

of electrical power accounting for the constraint dealing with limitation of 
pollutant released at the process outlet. Limitation of this strategy was concerned with the impossibilities to control overheating and to handle relatively strong stochastic variations of the inlet concentration, which is addressed here.

\subsection{LQR formulation}

The above control strategies are each based on a linear model obtained from the linearization of the nonlinear system (7) around a nominal steady state that we will define below. Obviously, the choice of the steady state around which the system is linearized is important for the control performance. Based on this remark, the nominal steady states can be calculated by solving the algebraic linear equation corresponding to $(7)$ in which $\dot{X}^{i}=0$. We proceed as follows:

- At the lower limit, we take the following values: $\Delta T_{a d}^{0}=0 K, \alpha^{0}=0.95$, $T_{s}^{0}\left(x_{101}\right)=450 K$. We deduce $Q_{j}^{0}=500 \mathrm{~W}$ and the steady state profile $Z^{0}$ (see Figure 4$)$.

$Z^{0}=\left(\begin{array}{c}Z^{10} \\ Z^{20} \\ Z^{30}\end{array}\right)=\left(\begin{array}{c}X^{10} \\ X^{20} \\ X^{30}\end{array}\right)$

- Similarly, at the higher limit: $\Delta T_{a d}^{1}=115 K, T_{s}^{1}\left(x_{101}\right)=600 K, Q_{j}^{1}=0 \mathrm{~W}$.

We deduce $\alpha^{1}=0.75$ and the corresponding steady state profile $Z^{1}$ (see 
Figure 4). $Z^{1}=\left(\begin{array}{l}Z^{11} \\ Z^{21} \\ Z^{31}\end{array}\right)==\left(\begin{array}{l}X^{11} \\ X^{21} \\ X^{31}\end{array}\right)$

Both systems are summarized in the following fashion:

$$
\left\{\begin{array}{l}
\Delta \dot{X}^{1}(t)=A^{1}\left(\alpha^{i}\right) \Delta X^{1}(t)+\left.\frac{\partial A^{1}(\alpha)}{\partial \alpha}\right|_{\alpha^{i}} Z^{1 i} \Delta \alpha+G^{1}\left(\alpha^{i}\right) \Delta X_{1}^{2}(t)+\left.\frac{\partial G^{1}(\alpha)}{\partial \alpha}\right|_{\alpha^{i}} Z^{2 i} \Delta \alpha \\
+\left.\frac{\partial d^{1}(\alpha)}{\partial \alpha}\right|_{\alpha^{i}} T_{0}(t) \Delta \alpha \\
\Delta \dot{X}^{2}(t)=A^{2}\left(\alpha^{i}\right) \Delta X^{2}(t)+\left.\frac{\partial A^{2}(\alpha)}{\partial \alpha}\right|_{\alpha^{i}} Z^{2 i} \Delta \alpha+G_{1}^{2}\left(\alpha^{i}\right) \Delta X_{100}^{1}(t)+G_{2}^{2}\left(\alpha^{i}\right) \Delta X_{1}^{3}(t) \\
+\left.\frac{\partial G_{1}^{2}(\alpha)}{\partial \alpha}\right|_{\alpha^{i}} Z_{100}^{1 i} \Delta \alpha+\left.\frac{\partial G_{2}^{2}(\alpha)}{\partial \alpha}\right|_{\alpha^{i}} Z_{1}^{3 i} \Delta \alpha+\left.\frac{\partial d^{2}(\alpha)}{\partial \alpha}\right|_{\alpha^{i}} \Delta T_{a d}^{i} \Delta \alpha+d^{2}\left(\alpha^{i}\right) \Delta \Delta T_{a d}(t)(10) \\
\Delta \dot{X}^{3}(t)=A^{3}\left(\alpha^{i}\right) \Delta X^{3}(t)+\left.\frac{\partial A^{3}(\alpha)}{\partial \alpha}\right|_{\alpha^{i}} Z^{3 i} \Delta \alpha+G^{3}\left(\alpha^{i}\right) \Delta X^{2}(t)+\left.\frac{\partial G^{3}(\alpha)}{\partial \alpha}\right|_{\alpha^{i}} Z^{2 i} \Delta \alpha \\
+\left.\frac{\partial B^{3}(\alpha)}{\partial \alpha}\right|_{\alpha^{i}} Q_{j}^{i} \Delta \alpha+B^{3}\left(\alpha^{i}\right) \Delta Q_{j}+\left.\frac{\partial d_{1}^{3}(\alpha)}{\partial \alpha}\right|_{\alpha^{i}} \Delta T_{a d}^{i} \Delta \alpha \\
+\left.\frac{\partial d_{2}^{3}(\alpha)}{\partial \alpha}\right|_{\alpha^{i}} T_{0}(t) \Delta \alpha+d_{1}^{3}\left(\alpha^{i}\right) \Delta \Delta T_{a d}(t)
\end{array}\right.
$$

Where $\Delta X^{j}=X^{j}-Z^{j i}$ with $j=1,2,3$ and $i=0$ or 1 . The above linearization is achieved around each steady state $Z^{i}=\left(\begin{array}{c}Z^{1 i} \\ Z^{2 i} \\ Z^{3 i}\end{array}\right)=\left(\begin{array}{c}X^{1 i} \\ X^{2 i} \\ X^{3 i}\end{array}\right)$ with, $i=0,1$. Both systems are summarized as:

$$
\Delta \dot{X}(t)=A\left(\alpha^{i}\right) \Delta X(t)+B_{1}^{i} \Delta \alpha(t)+B_{2}^{i} \Delta Q_{j}(t)
$$


Remark 4.1 A discussion often arises at this point: what is the need to develop an accurate and complex nonlinear model (like here) if a linearized model is finally used for the control synthesis? It is known that the advantage is that the on-line computational effort is less important, while the drawbacks are the linearization errors. In the case of the RFR, the linearization errors around the nominal steady state have been shown to be not very important: indeed, the accurate nonlinear model (1-5) is a nonlinear model with respect to the input, but a linear model with respect to the state. Therefore, errors due to the linearization are very reasonable.

The LQR control aims binding the catalytic temperature in the optimal operating conditions defined by the temperature envelope. Therefore, two cost functions are considered, which allows diagonalizing the system. Indeed, it seems natural to avoid both heating and cooling together. With this linear system, the control strategy is defined by the two following cost functions:

- When the pollutant concentration is too low, the controller aims to tune the internal heating power such that the temperature is kept over a lower bound threshold. In the LQR stategy, this aims to minimize the following cost:

$$
\int_{0}^{\infty}\left(p_{1}^{0}\left(\Delta X^{2}(t)\right)^{2}+p_{2}^{0}\left(\Delta Q_{j}(t)\right)^{2}\right) d t
$$

- When the pollutant concentration is too high, the controller aims to tune the fresh air dilution such that the temperature is kept below an upper 
bound threshold. In the LQR strategy, this aims to minimize the following cost:

$$
\int_{0}^{\infty}\left(p_{1}^{1}\left(\Delta X^{2}(t)\right)^{2}+p_{2}^{1}(\Delta \alpha(t))^{2}\right) d t
$$

In both cases, the control obtained from the minimization of these cost functions is a linear feedback of the form $\Delta Q_{j}(t)=-F^{0} \Delta X$ or $\Delta \alpha(t)=-F^{1} \Delta X$ with $\Delta X=X_{e}(t)-Z^{i}$ where $(i=0,1)$ and $X_{e}(t)$ is the estimate state given by the observer. The gains of the feedback matrix are obtained from the solution of the classical algebraic Riccati equation:

$$
\left\{\begin{array}{l}
F^{0}=\left(p_{2}^{0}\right)^{-1}\left(B_{2}^{0}\right)^{T} S^{0} \\
S^{0} A\left(\alpha^{0}\right)+\left(A\left(\alpha^{0}\right)\right)^{T} S^{0}+C^{T} C-S^{0} B_{2}^{0}\left(p_{2}^{0}\right)^{-1}\left(B_{2}^{0}\right)^{T} S^{0}=0 \\
F^{1}=\left(p_{2}^{1}\right)^{-1}\left(B_{1}^{1}\right)^{T} S^{1} \\
S^{1} A\left(\alpha^{1}\right)+\left(A\left(\alpha^{1}\right)\right)^{T} S^{1}+C^{T} C-S^{1} B_{1}^{1}\left(p_{2}^{1}\right)^{-1}\left(B_{1}^{1}\right)^{T} S^{1}=0
\end{array}\right.
$$

where $C=\left[C_{1} \ldots C_{100} C_{101} \ldots C_{200}\right]$ with $\left[C_{1} \ldots C_{100}\right]=[0 \ldots 0]$ and $\left[C_{101} \ldots C_{200}\right]=$ $\left[\begin{array}{llll}1 & 0 & \ldots & 0\end{array}\right]$ 


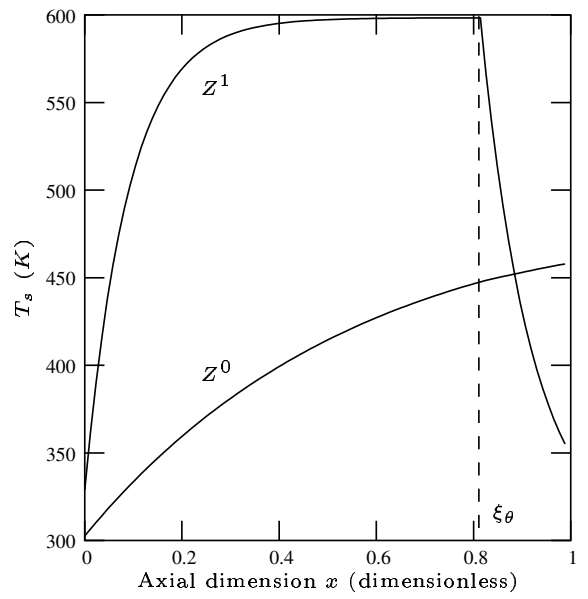

Fig. 4. LQR: Nominal temperature profiles in high and low temperature operations. 


\subsection{MPC formulation}

From a practical point of view, one of the drawbacks of the MPC is the computational time aspect, especially when the model becomes more complex and more accurate, like the model of the RFR model presented here. Indeed, the model is intended to predict the future dynamic behaviour of the process output over a finite prediction horizon and has to be solved during the on-line constrained optimization problem resolution. The method used here to reduce this computational time [13] is now reminded. The idea is to use two models on-line: the nonlinear parabolic PDE model (1-5) solved off-line combined with a linearized time-varying PDE model (11) solved on-line during the optimization task. The use of the IMC structure allows using less accurate (hence less time consuming) finite dimensional approximation of the linearized timevarying PDE model (11) hence introducing robustness with respect to model approximation [14]. Indeed, for the model approximation, two tuning strategies are possible for the number of nodes used in the finite difference method: First possibility, the number of nodes is tuned to a "small" value ("small" with respect to the large number of nodes to find an accurate approximation of the nonlinear parabolic PDE system solution). In this case, less time is required for the model resolution and more time can be spent for the optimization task. But since the MPC formulation is an open loop predictive optimization procedure, large errors due to the PDE model solution approximation used in the optimization task may degrade closed-loop control results. The second possibility for the tuning is to increase the number of nodes to find a more accurate approximated solution of the PDE system. This leads to increase the time needed to solve the model and to decrease the time dedicated to 
the optimization task resolution. The drawback is that the optimizer may not have the time to find a solution during the sampling time. Clearly speaking, a trade-off exists for this tuning: even if it leads to a loss of accuracy in open loop input output behaviour, it allows reaching the closed-loop performances specified while providing a less time-consuming resolution task [14].

Here, two types of constraints are handled: hard constraints are those who should never be violated while soft constraints may sometimes be violated [34]. Hard input constraints (magnitude and velocity constraints for the manipulated variables (MVs)) are handled through a transformation method: it guarantees that the actions always respect these constraints. Soft output constraints (constraints on the controlled variables $(\mathrm{CVs})$ ) are accounted for in an exterior penalty method [19]: this allows handling for unfeasibility issue that main happened during the constrained optimization task resolution.

Finally, the control problem is formulated in the MPC framework as:

$$
\left\{\begin{array}{l}
\min _{Q_{j}(k), \alpha(k)} J\left(Q_{j}(k), \alpha(k)\right)=\left(w_{Q}\left[\frac{Q_{j}(k)-Q_{j \cdot \min }}{Q_{j \cdot \max }-Q_{j \cdot \min }}\right]^{2}+w_{\alpha}\left[\frac{\alpha(k)-\alpha_{\max }}{\alpha_{\max }-\alpha_{\min }}\right]^{2}\right) \\
\text { with constraints on MVs magnitude: } \\
Q_{j \cdot \min } \leq Q_{j}(j) \leq Q_{j \cdot \max } \quad \forall j \in J_{0}^{h_{p}-1}=\left\{k, k+h_{p}-1\right\} \\
\alpha_{\min }<\alpha(j) \leq \alpha_{\max } \quad \forall j \in J_{0}^{h_{p}-1} \\
\text { with } 2 \text { constraints on the CVs: } \\
T_{s . \min } \leq T_{s}\left(x_{101}, j\right) \quad\left(\text { with } j \in\left\{k+h p_{1}^{i}, k+h p_{1}^{f}\right\}\right) \\
T_{s}\left(x_{101}, j\right) \leq T_{s . \max } \quad\left(\text { with } j \in\left\{k+h p_{2}^{i}, k+h p_{2}^{f}\right\}\right)
\end{array}\right.
$$


where $k$ is the actual discrete time index, $j$ is the discrete time index. The tuning parameters are the horizons: $h p_{1}^{i}$ and $h p_{1}^{f}$ (resp. $h p_{2}^{i}$ and $h p_{2}^{f}$ ) are the initial and future prediction horizons describing the lower (resp. upper) temperature constraint whereas $h_{p}=\max \left(h p_{1}^{f}, h p_{2}^{f}\right) . w_{Q}$ and $w_{\alpha}$ are positive weights. In the meantime, in order to decrease the computational burden, the tuning of the control horizon is one. Even if it is true that this tuning reduces strongly the degrees of freedom to solve the optimization problem, it allows decreasing the computational time, which is an important issue here. Indeed, the control horizon is the number of arguments that characterizes the sequence of future control moves involved in the optimization task: the closed-loop performances improve when the control horizon increases but at the expense of increasing the computational burden [34].

\section{Simulation results}

\section{$5.1 L Q R$}

By use of the observer, full states of the system can be estimated in real time, which makes implementation of LQR possible. For the LQR control, we have: $-\Delta \alpha(t)=-F^{1} \Delta X$ and $\Delta Q_{j}(t)=-F^{0} \Delta X(t)$ with $\Delta X(t)=X_{e}(t)-Z^{i}(t)$ (where $X_{e}(t)$ is the estimated state).

Since the feedback gain can be determined off-line, the time used to compute control actions online is negligible. The weights are tuned as follows: $P_{1}^{0}=$ $P_{1}^{1}=1, P_{2}^{0}=5 \times 10^{-4}$ and $P_{2}^{1}=500$. Simulations results show that, in spite of the steep changes in feed concentration (Figure 2), the temperature at the inlet of catalytic zone is tightly controlled between the two limits (Figure 5). 
The LQR correctly tune both manipulated variables: indeed, between $500 s$ and $1550 s$, the lean feed leads to decrease the temperature inside the reactor. LQR tunes the internal heating (Figure 6) to a value needed to keep the temperature above the extinction temperature. In the meantime, no dilution is taking place (Figure 7). After 1550s, rich feed induces an increase of temperature inside the reactor. LQR tunes the dilution rate (Figure 7) such that the temperature is maintained below the maximum temperature (Figure 5). There is no heating (Figure 6). At the end of the run, the average of electrical power $\bar{Q}_{j}$ is $83.4 \mathrm{~W}$, the average of dilution rate $\bar{\alpha}$ is 0.894 , whereas constraints are always satisfied. 


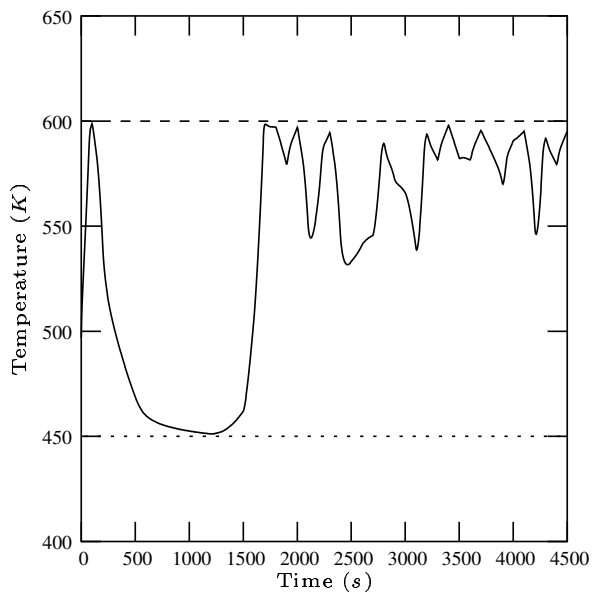

Fig. 5. LQR: Hot spot temperature.

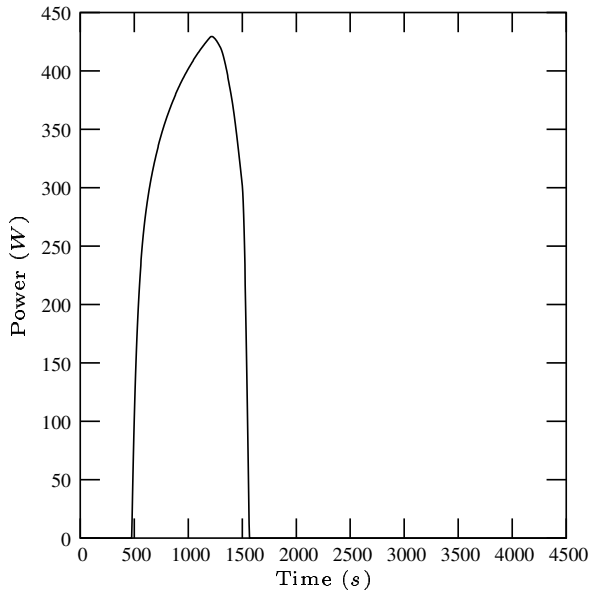

Fig. 6. LQR: Heating power.

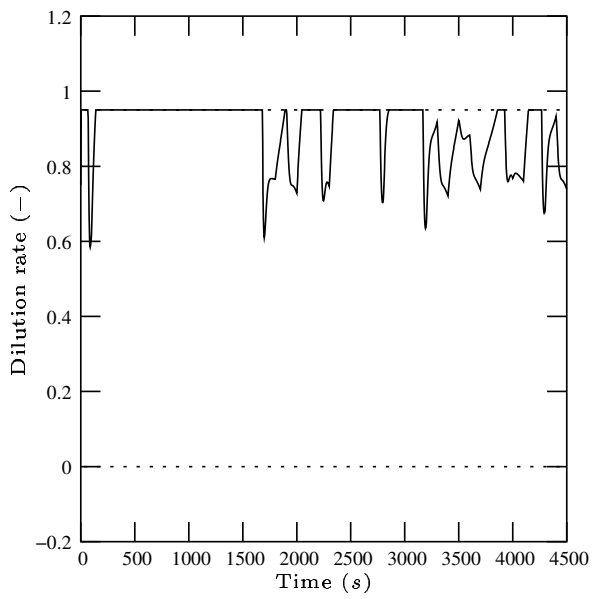

Fig. 7. LQR: Dilution rate. 


\section{2 $M P C$}

Simulation runs are now discussed for the use of the proposed MPC strategy.

They allow seeing different closed-loop behaviours according to:

- the tuning of the controller, i.e. the 2 initial receding horizons and the 2 final horizons,

- the use of the observer into the control loop.

\subsubsection{Tuning of the controller}

It is clear that the tuning of the controller parameters directly influences the constrained optimization problem and therefore closed-loop control results. Moreover, this constrained optimization problem uses the estimation of the input disturbance (the adiabatic temperature rise $\Delta T_{a d}$ ) which has an evident impact over the closed-loop performances (especially the constraints satisfaction). Regarding the impact of the input disturbance over the optimization task and the constraints satisfaction required, some guidelines for the tuning of the controller parameters are first given in the table 1.

It clearly underlines that the tuning will be uneasy due to the impact of the input disturbance over the closed-loop performances and due to the non minimum phase behaviour of the temperature as well. Indeed, according to the tuning of the manipulated variables, the temperature inside the reactor may feature a non-minimum phase behaviour, as it can be seen on Figure 8: it depicts the evolution in time of the sensitivity of the temperature with respect to the dilution rate at two different locations in the reactor. The reason of this non minimum phase behaviour is not clear for the moment. But one can see 
that when the dilution action is increased, two phenomena occur: first, as expected, the action of dilution allows decreasing the temperature inside the reactor (the sensitivity takes positive final values). In the meantime, the flow rate inside the thermal monolith is decreased hence increasing heat exchange between the gas and the solid part (the sensitivity takes negative values). Therefore, before the expected influence of dilution action appears, dilution action is acting as a heating action. 


\begin{tabular}{|c|c|c|}
\hline & $\begin{array}{l}\text { Impact of } \Delta T_{a d} \text { during the opti- } \\
\text { mization task according to .... }\end{array}$ & $\begin{array}{l}\text { Constraints satisfaction during the } \\
\text { optimization task according to ... }\end{array}$ \\
\hline $\begin{array}{l}. . \text { the tuning of } \\
\text { the initial horizon }\end{array}$ & $\begin{array}{l}\text { If some constraints are currently } \\
\text { saturated, they may no more be sat- } \\
\text { isfied in the very close future due to } \\
\text { possible strong variations of } \Delta T_{a d} \text {. } \\
\text { Conclusion: the smallest initial pre- } \\
\text { diction horizon is required. }\end{array}$ & $\begin{array}{l}\text { The cooling action creates a non- } \\
\text { minimum phase behaviour in the } \\
\text { temperature involved in output con- } \\
\text { straints. Therefore, initial predic- } \\
\text { tion horizon has to be tuned suf- } \\
\text { ficiently large such that this be- } \\
\text { haviour is not accounted for into } \\
\text { the controller, especially when con- } \\
\text { straints are saturated. Conclusion: } \\
\text { a sufficiently large initial prediction } \\
\text { horizon is required. }\end{array}$ \\
\hline $\begin{array}{l}\ldots \text { the tuning of } \\
\text { the final horizon } \\
\text { prediction }\end{array}$ & $\begin{array}{l}\text { Since } \Delta T_{a d} \text { is used in the prediction } \\
\text { framework, is strongly stochastic } \\
\text { and has a large impact over closed- } \\
\text { loop performances, large prediction } \\
\text { horizons will forecast an uncertain } \\
\text { future. Conclusion: the smallest fi- } \\
\text { nal prediction horizon is required. }\end{array}$ & $\begin{array}{l}\text { The dynamic of the temperature in- } \\
\text { volved in the output constraints is } \\
\text { relatively low and has to be ac- } \\
\text { counted for into the constrained } \\
\text { optimization problem. Conclusion: } \\
\text { a sufficiently large final prediction } \\
\text { horizon is required. }\end{array}$ \\
\hline
\end{tabular}

Table 1

Guidelines for the tuning of the initial and final prediction horizons. 


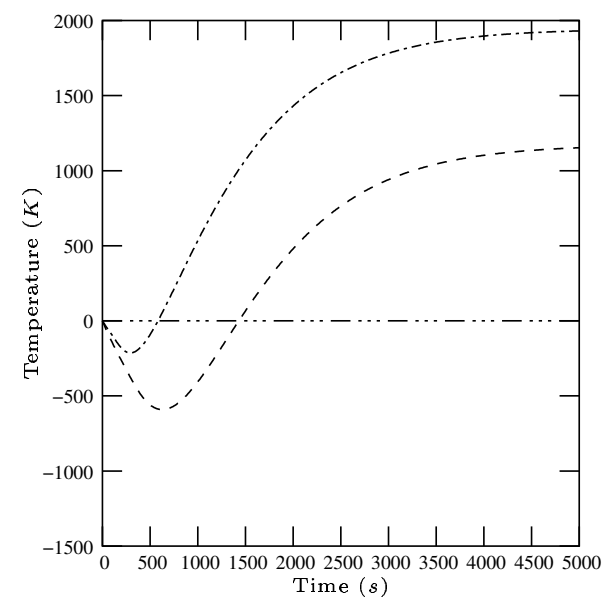

Fig. 8. Time evolution of 2 distributed sensitivities of the temperature with respect to the dilution rate. 


\subsubsection{Correct tuning of the controller}

In run \#778, the tuning of the controller parameter are the following one:

$$
h p_{1}^{i}=2, h p_{1}^{f}=4, h p_{2}^{i}=1, h p_{2}^{f}=12
$$

In this run, one assumes that the estimation of the input disturbance $\Delta T_{a d}$ (Figure 2) is fed into the controller. This run shows that this controller's tuning allow to satisfy the constraints at any time (Figure 9), which is the most important point required by the constrained optimization problem. One can see 2 different time intervals:

- For $0<t<1300 s, \Delta T_{a d}$ is small (Figure 2) and extinction of the process is avoided feeding electrical power into the reactor (Figure 10). In the meantime, there is no cooling action (except a peak at the beginning) and the maximum amount of gas is therefore treated (Figure 11) as expected in these conditions.

- When $t>1500 s, \Delta T_{a d}$ becomes important and overheating of the process is avoided (see the upper bound constraint on Figure 9) due to the correct use of cooling action (Figure 11). The drawback is that the controller may sometimes require both heating and cooling actions at the same time (Figure 10 and 11), which should not happen: optimization should be improved to avoid this issue. 


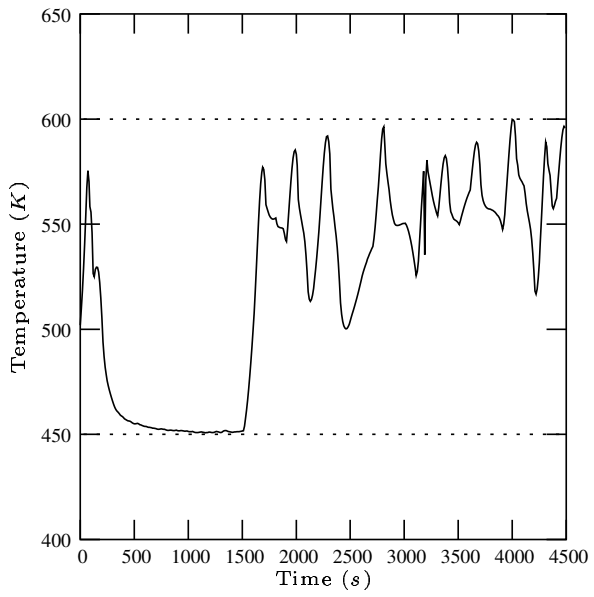

Fig. 9. MPC, run \#778: Hot spot temperature.

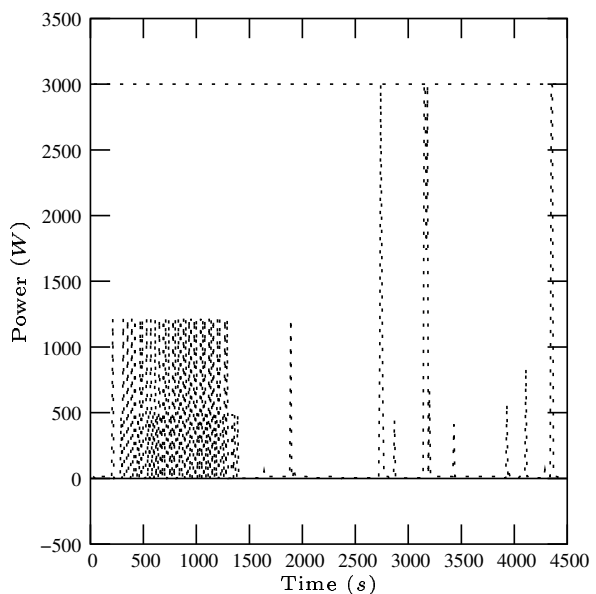

Fig. 10. MPC, run \#778: Heating power.

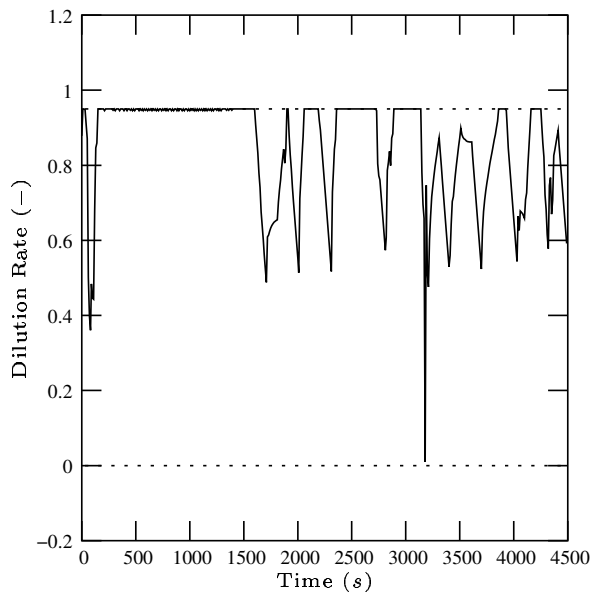

Fig. 11. MPC, run \#778: Dilution rate. 


\subsubsection{Bad tuning of the controller}

In the previous simulation, constraints were always satisfied. In the meantime, cooling and heating action were sometimes acting at the same time, which should not happened with the RFR. Therefore, in order to improve the tuning of the control actions, the controller parameters were retuned as follow in run \#787 accounting for the remarks made in table 1:

$$
h p_{1}^{i}=1, h p_{1}^{f}=3, h p_{2}^{i}=8, h p_{2}^{f}=8
$$

In this run, one assumes that the estimation of the input disturbance $\Delta T_{a d}$ (Figure 2) is fed into the controller. The most important changes between runs \#787 and \#778 is dealing with $h p_{2}^{i}$. Its tuning allows accounting more correctly for the temperature's NMP behaviour: both cooling and heating are not acting at the same time after 1500 s (Figure 13 and 14). The NMP behaviour is much less influencing the optimization task. But the drawback of such tuning is evident over the constraints (Figure 12): as predicted in table 1 , the temperature is going beyond the maximum threshold due to the strong impact of $\Delta T_{a d}$ and since near future time is no more accounted for into the constrained optimization task. Therefore, the controller reacts only after constraint's violation. 


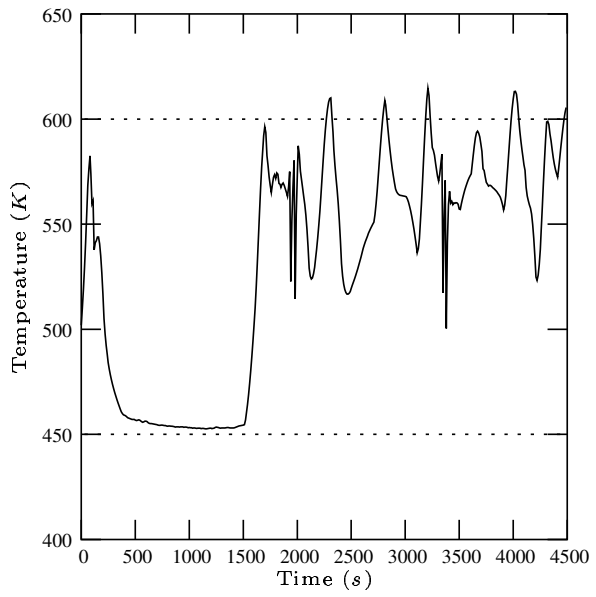

Fig. 12. MPC, run \#787: Hot spot temperature.

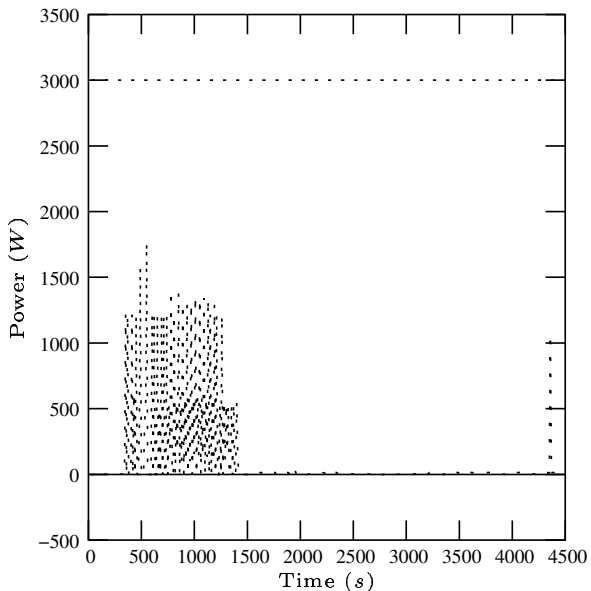

Fig. 13. MPC, run \#787: Heating power.

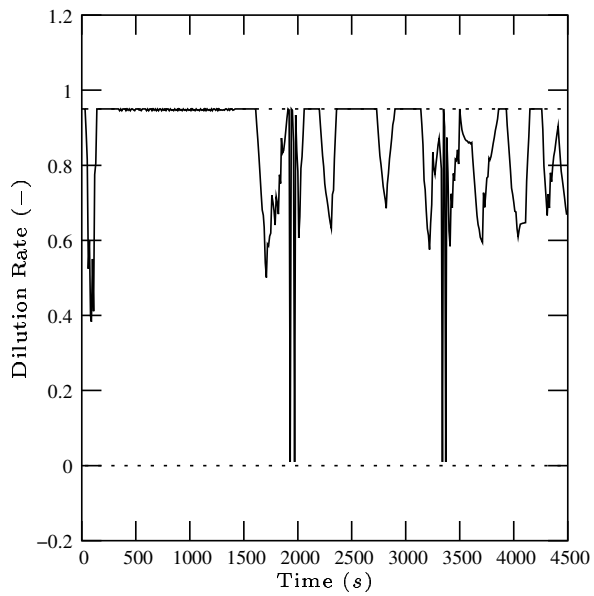

Fig. 14. MPC, run \#787: Dilution rate. 


\subsubsection{Comparison of the two previous tunings of the controller}

Finally, even if the rules given in table 1 are very important to correctly tune the controller, the MPC tuning is clearly an uneasy task. For such process, the MPC may be tuned according to two different strategies:

- run \#787: the output constraints may not be satisfied at any time (soft constraints): this leads the controller to a correct decoupling of the two actions while the output constraints are not always satisfied.

- run \#778: the output constraints have to be satisfied at any time (hard constraints): this leads the controller to tune both actions such that the output constraints are always satisfied while the two actions may sometimes act at the same time.

Here, run \#778, even if leading to less interesting results than run \#787 in term on control actions, features the best controller tuning for this constrained control problem which is very sensitive with respect to the stochastic input disturbance $\Delta T_{a d}$. At the end of the run \#778, overall optimization is interesting: the average of electrical power $\bar{Q}_{j}$ is $274.6 \mathrm{~W}$, the average of dilution rate $\bar{\alpha}$ is 0.849 , whereas constraints are always satisfied.

\subsubsection{Need for the observer}

Previous runs are assuming that the measure of the stochastic input disturbance $\Delta T_{a d}$ is available. One present here results obtained in run \#798, which is similar to run \#778, but where no estimation of $\Delta T_{a d}$ is used by the controller. It clearly shows the strong need for the use of the observer since both output constraints are not always satisfied (Figure 15). This also clearly under- 
lines, as expected, the strong impact of the stochastic input disturbance over closed-loop control results and the difficulty to handle it in the optimization task. 


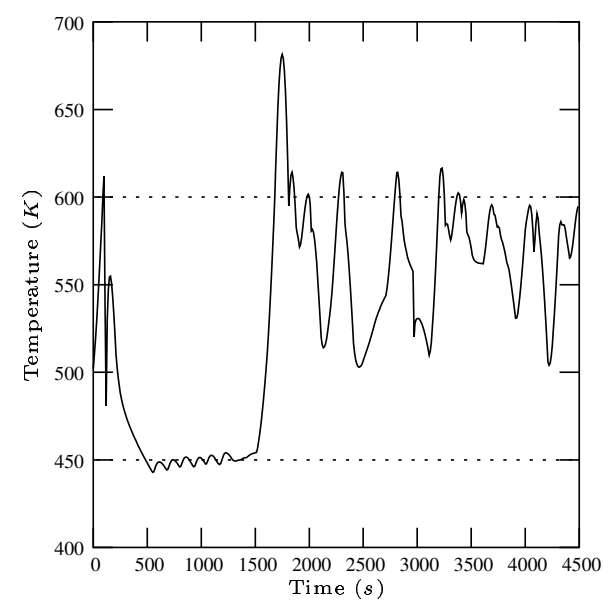

Fig. 15. MPC, run \#798: Hot spot temperature. 


\subsection{Comparison between $L Q R$ and $M P C$ results}

With both control strategies, the temperature can be maintained into the specified temperature envelope, in spite of large input disturbance due to the feed concentration. Concerning the optimization performances, LQR leads to better results than $\mathrm{MPC}: \bar{Q}_{j}=83.4 \mathrm{~W}$ and $\bar{\alpha}=0.894$ for $\mathrm{LQR}, \bar{Q}_{j}=274.6 \mathrm{~W}$ and $\bar{\alpha}=0.849$ for MPC. LQR is therefore more interesting since it requires less heating action while treating more gas. This difference is mostly due to the impact of the stochastic variations of the input disturbance over MPC. Indeed, the estimation of the disturbance $\Delta T_{a d}$ is directly used in the MPC, where it is assumed constant in the future. This forces the MPC to over evaluate the need for heating and cooling. In the meantime, such assumption is not required by the LQR which makes it more interesting for control purpose, even if the estimation of $\Delta T_{a d}$ is not directly used by the LQR. In the meantime, a switching control structure is used for the LQR: therefore, heating and cooling action are decoupled and can not acting at the same time as expected. The drawback is that it introduces a severe nonlinearity for stability analysis. Concerning the MPC approach, most of the time, cooling and heating are not acting at the same time. But due to the large impact of the input disturbance, due to the non minimum phase behaviour of the process, due to the prediction aspect and due to the uneasy task of horizons tuning, heating and cooling actions may be sometimes required at the same time. This is clearly an unexpected behaviour which degrades the closed-loop performance. In the meantime, the proposed MPC approach is a general framework for multivariable control problem whereas the LQR assumes decoupling. Moreover, constraints are explicitly accounted for in the MPC formulation whereas they 
are handled in a logical decision level in the LQR.

\section{Conclusion}

This paper is dealing with the performances comparison of two multivariable observer based controllers of a catalytic RFR used to decrease noxious VOC amount released in the atmosphere. The complexity of this process includes distributed aspect, nonlinear dynamic behaviour and periodic reversing of the circulation of gas. Until now, very few papers have dealt with the control of such process, especially the multivariable optimal control. In this paper, we compare a LQR and a MPC both based on a high-gain observer. The new key control issue tackled here is to confine the hot spot temperature within two temperature limits, in order to ensure complete conversion of the pollutant and to prevent catalyst overheating, while optimizing the control actions. In spite of a large input disturbance due to the stochastic variations of the feed concentration, both observer based controllers are very robust since the temperature can be maintained inside the specified temperature envelope. The controlled RFR may therefore be used under various operating conditions, which is very interesting for the industrial use. Since the LQR directly uses the state estimation (whereas the MPC directly uses the input disturbance estimation), the large input disturbance has less impact over LQR performances than MPC performances. Therefore, the LQR leads to a better optimal operation of the RFR than MPC: less heating action is required while treating more gas. In the meantime, LQR strategy is based on two decoupled optimization tasks and a logical decision level whereas MPC is based on a general multivariable optimization task where constraints are explicitly accounted for. This 
makes MPC more suitable for general multivariable control problems. It is also shown how the MPC tuning is an uneasy task due to the process behaviour, the optimization problem and the impact of the input disturbance.

\section{References}

[1] Allgöwer, F., Badgwell, T.A., Qin, J.S., Rawlings, J.B., \& Wright, S.J. (1999). Nonlinear predictive control and moving horizon estimation. In P.M. Frank (Ed.) Advances in control. Highlights of ECC'99, Springer, Berlin.

[2] Armaou, A., \& Christofides, P.D. (2002). Dynamic optimization of dissipative PDE systems using nonlinear order reduction. Chem. Eng. Sci., 57(24), 50835114.

[3] Balas, M.J. (1998). Stable feedback control of linear distributed parameter systems: time and frequency domain conditions. J. Math. Anal. Appl., 225(1), 144-167.

[4] Balakotaiah, V., \& Dommeti, S.M.S (1999). Effective models for packed-bed catalytic reactors. Chem. Eng. Sci, 54, 1621.

[5] Bornard., G, \& Hammouri., H. (1991). A high-gain observer for a class of uniformly observable systems. Proc. of the $30^{\text {th }}$ IEEE Conference on Decision and Control, Brighton, England.

[6] Budman, H., Kzyonsek, M., \& Silverston, P. (1996). Control of a nonadiabatic packed bed reactor under periodic flow reversal. Canadian J. of Chem. Eng., 74, 751-759. 
[7] Christofides, P.D., \& Daoutidis, P. (1997). Finite-dimensional control of parabolic PDE systems using approximate inertial manifolds. J. Math. Anal. Appl., 216(2), 398-420.

[8] Christofides, P.D. (1998). Robust control of hyperbolic PDE systems. Chem. Eng. Sci., 53(16), 85-105.

[9] Cittadini, M., Vanni, M., Barresi, A.A., \& Baldi, G. (2001). Simplified procedure for design of catalytic combustors with periodic flow reversal Chem. Eng. Process., 40(3), 255-262.

[10] Cunill, F., Van de Beld, L., \& Westerterp, K.R. (1997). Catalytic combustion of very lean mixtures in a reverse flow reactor using an internal electrical heater. Ind. Eng. Chem. Res., 36, 4198-4206.

[11] Deza., F, Busvelle., E, \& Gauthier, .J.P. (1992). High-gain estimation for nonlinear systems. Syst. E Contr. Letters, 18, 295.

[12] Dufour, P., Couenne, F., \& Touré, Y. (2003). Model predictive control of a catalytic reverse flow reactor. Special issue of IEEE Trans. on Control Syst. Technol. on Control of Industrial Spatially Distributed Parameter Processes, 11(5), 705-714.

[13] Dufour P., Touré, Y., Blanc D., \& Laurent, P. (2003) On nonlinear distributed parameter model predictive control strategy: on-line calculation time reduction and application to an experimental drying process. Comput. \& Chem. Eng., 27(11), 1533-1542.

[14] Dufour P., \& Touré, Y., (in press) Multivariable model predictive control of a 
catalytic reverser flow reactor. Comput. \& Chem. Eng., in press.

[15] Eaton, J.W., \& Rawlings, J.B. (1990). Feedback control of chemical processes using on-line optimization techniques. Comput. \& Chem. Eng., 14(4-5), 469-479.

[16] Edouard, D., Hammouri, H., \& Schweich, D. (2004). Observer design for reverse flow reactor. AIChE J., 50(9), in press.

[17] El-Farra, N.H., Armaou, A., \& Christofides, P.D. (2003). Analysis and control of parabolic PDE systems with input constraints. Automatica, 39(4), 715-725.

[18] Farza., M, Hammouri., H, Busavon., K. (1998). A simple observer for a class of nonlinear systems. Appl. Math. Letters, 11, 27.

[19] Fletcher, R. (1987). Practical methods of optimization. John Wiley and Sons.

[20] Gauthier. J.P, Hammouri. H., \& Othman. S. (1992). A simple observer for nonlinear systems, application to bioreactors, IEEE Trans. Automat. Control, 37,875 .

[21] Godasi, S., Karakas, A., \& Palazoglu, A. (2002). Control of nonlinear distributed parameter processes using symmetry groups and invariance conditions. Comput. G Chem. Eng., 26(7-8), 1023-1036.

[22] Hoo, K.A., \& Zheng, D. (2001). Low-order control-relevant models for a class of distributed parameter systems. Chem. Eng. Sci., 56(23), 6683-6710.

[23] Irizarry-Rivera R., \& Seider, W.D. (1997). Model-predictive control of the Czochralski crystallization process Part I. Conduction-dominated melt. J. of Crystal Growth, 178(4), 593-611. 
[24] Keith, J.M. (2003). Controlling reverse-flow reactors via multiscale transient thermal dispersion. Adv. in Environm. Res., 7(2), 521-535.

[25] Levine, W.S. (1996). The control handbook. CRC and IEEE Press.

[26] Marquis, P., \& Broustail, J. (1988). SMOC, a bridge between state space and model predictive controllers: application to the automation of a hydrotreating unit. Proc. of the IFAC Workshop on Model Based Process Control, 37-43.

[27] Mayne, D.Q., Rawlings, J.B., Rao, C.V., \& Scokaert, P.O.M. (2000). Constrained model predictive control: stability and optimality. Automatica, $36(6), 789-814$.

[28] Morari, M., \& Zafiriou, E. (1989). Robust process control. Dunod.

[29] Morari, M., \& Lee, J.H. (1999). Model predictive control: past, present and future. Comput. \& Chem. Eng., 23(4-5), 667-682.

[30] Nieken, U., Kolios, G., \& Eigenberger, G. (1995). Control of the ignited steady state in authothermal fixed-bed reactors for catalytic combustion. Chem. Eng. Sci., 49(24), 5507-5518.

[31] Nieken, U., Kolios, G., \& Eigenberger, G. (1995). Limiting cases and approximate solutions for fixed-bed reactors with periodic flow reversal. AIChE $J ., 41(8), 1915-1925$.

[32] Palazoglu, A., \& Karakas, A. (2000). Control of nonlinear distributed parameter systems using generalized invariants. Automatica, 36(5), 697-703.

[33] Patwardhan, A.A., Wright, G.T., \& Edgar, T.F. (1992). Nonlinear modelpredictive control of distributed-parameter systems. Chem. Eng. Sci., 47(4), 
721-735.

[34] Qin, S.J., \& Badgwell, T.A. (2003). A survey of industrial model predictive control technology. Control Eng. Pract., 11(7), 733-764.

[35] Ramdani, K., Pontier, R., \& Schweich, D. (2001). Reverse low reactor at short switching periods for VOC combustion. Chem. Eng. Sci., 56(4), 1531-1539.

[36] Rawlings, J.B (2000). Tutorial overview of model predictive control. IEEE Control Syst., 20(3), 38-52.

[37] Touré, Y., Biston, J., \& Gilles, G. (1994). Modeling of a distributed parameter process with a variable boundary: application to its control. Chem. Eng. Sci., $49(1), 61-73$.

[38] Touré, Y., \& Josserand, L. (1997). An extension of IMC to boundary control of distributed parameter systems. Proc. of the IEEE International Conference on Systems, Man and Cybernetics-CCS, Orlando, USA, 3, 2426-2431.

[39] Vortmeyer, D., \& Schaefer, R. J. (1974). Equivalence of one- and two-phase models for heat transfer processes in packed beds: one dimensional theory. Chem. Eng. Sc., 29(2), 485-491

[40] Zheng, D., \& Hoo, K.A. (2002). Low-order model identification for implementable control solutions of distributed parameter systems. Comput. $\mathscr{G}$ Chem. Eng., 26(7-8), 1049-1076.

[41] Zheng, D., \& Hoo, K.A. (2004). System identification and model-based control for distributed parameter systems. Comput. \& Chem. Eng., 28(8), 1361-1375. 


\section{$7 \quad$ Appendix}

\subsection{Matrices expression}

$A^{1}(t)=\left(\begin{array}{ccccc}a_{4}(t) & a_{1}(t) & 0 & \ldots & 0 \\ a_{3}(t) & a_{2}(t) & a_{1}(t) & & \vdots \\ 0 & \ddots & \ddots & \ddots & \vdots \\ \vdots & & \ddots & \ddots & a_{1}(t) \\ 0 & \ldots & 0 & a_{3}(t) & a_{2}(t)\end{array}\right), A^{2}(t)=a_{2 c}(t)$,

$A^{3}(t)=\left(\begin{array}{ccccc}a_{2}(t) & a_{1}(t) & 0 & \ldots & 0 \\ a_{3}(t) & a_{2}(t) & a_{1}(t) & & \vdots \\ 0 & \ddots & \ddots & \ddots & \vdots \\ \vdots & & \ddots & \ddots & a_{1}(t) \\ 0 & \cdots & 0 & a_{3}(t) & a_{5}(t)\end{array}\right)$.

$d^{1}(t)=\left(\begin{array}{c}b_{1}(t) \\ 0 \\ \vdots \\ 0\end{array}\right), d^{2}(t)=r(t)$, with $r(t)=\frac{P_{\theta} \psi\left(x_{101}\right)}{\tau}$,

$d_{1}^{3}(t)=\left(\begin{array}{c}\frac{P_{\theta} \psi\left(x_{102}\right)}{\tau} \\ \frac{P_{\theta} \psi\left(x_{103}\right)}{\tau} \\ \vdots \\ \frac{P_{\theta} \psi\left(x_{200}\right)}{\tau}\end{array}\right), d_{2}^{3}(t)=\left(\begin{array}{c}0 \\ 0 \\ \vdots \\ \\ b_{2}(t)\end{array}\right)$ 


$$
\begin{aligned}
G^{1}(t) & =\left(\begin{array}{c}
0 \\
\vdots \\
a_{1}(t)
\end{array}\right), G_{1}^{2}(t)=a_{3 c}(t), G_{2}^{2}(t)=a_{1}(t) \\
G^{3}(t) & =\left(\begin{array}{c}
a_{3}(t) \\
0 \\
\vdots \\
0
\end{array}\right) \cdot B^{3}(t)=\left(\begin{array}{c}
0 \\
\vdots \\
b_{3}(t)
\end{array}\right) .
\end{aligned}
$$

The coefficients $a_{i}, a_{i c}$ and $b_{i}$ are given by:

$$
\begin{aligned}
& a_{1}(t)=\frac{\lambda_{e f f 1}(t)}{\left(\delta x_{k}\right)^{2}}, a_{2}(t)=-2 \frac{\lambda_{e f f 1}(t)}{\left(\delta x_{k}\right)^{2}}+\frac{D(t)}{\delta x_{k}}, \quad a_{3}(t)=\frac{\lambda_{e f f 1}(t)}{\left(\delta x_{k}\right)^{2}}-\frac{D(t)}{\delta x_{k}}, \\
& a_{4}(t)=a_{2}(t)+a_{3}(t) \frac{\alpha(t)}{P_{\theta} \delta x_{1}} \frac{1}{1+\frac{\alpha(t)}{P_{\theta} \delta x_{1}}}, \\
& a_{5}(t)=a_{2}(t)+a_{1}(t) \frac{1+N^{\prime}+\alpha^{2}(t)}{P_{\theta} \delta x_{2}} \frac{1}{\left(1+N^{\prime}\right)\left(1+\frac{1}{P_{\theta} \delta x_{2}}\right)+\frac{\alpha^{2}(t)}{P_{\theta} \delta x_{2}}-\alpha(t)} . \\
& \text { and } a_{2 c}(t)=\left(\delta x_{1}+\delta x_{2}\right) \frac{\lambda_{e f f 1}(t)}{\delta x_{1}\left(\delta x_{2}\right)^{2}}+\frac{D(t)}{\delta x_{1}}, a_{3 c}(t)=\frac{\lambda_{e f f 1}(t)}{\left(\delta x_{1}\right)\left(\delta x_{2}\right)}-\frac{D(t)}{\delta x_{1}}, \\
& b_{1}(t)=a_{3}(t) \frac{1}{1+\frac{\alpha(t)}{P_{\theta} \delta x_{1}}}, \quad b_{2}(t)=a_{1}(t) \frac{1+N^{\prime}-\alpha(t)}{\left(1+N^{\prime}\right)\left(1+\frac{1}{P_{\theta} \delta x_{2}}\right)+\frac{\alpha^{2}(t)}{P_{\theta} \delta x_{2}}-\alpha(t)}
\end{aligned}
$$

and $b_{3}(t)=a_{1} \frac{1}{\rho_{0} u_{V_{0}} c_{p m g} S} \frac{1}{\left(1+N^{\prime}\right)\left(1+\frac{1}{P_{\theta} \delta x_{2}}\right)+\frac{\alpha^{2}(t)}{P_{\theta} \delta x_{2}}-\alpha(t)}$

with $D(t)=\frac{1-\alpha(t)}{2 \tau}, \lambda_{e f f 1}(t)=\frac{1}{\tau P_{a x \theta}}+\frac{1+\alpha^{2}(t)}{2 P_{\theta} \tau}$

and $\delta x_{k}=\delta x_{1}=8.3651 .10^{-3}$ for $k=1$ (inert monolith), $\delta x_{k}=\delta x_{2}=$ $1.5513 .10^{-3}$ ) for $k=2$ (catalytic monolith). 
$L^{1}(t)=a_{1}(t) \Theta_{1} K^{1}, L^{2}(t)=\Lambda(t) \Theta_{2} K^{2}$ and $L^{3}(t)=a_{3}(t) \Theta_{3} K^{3} J$

where, $K^{1}=\left(\begin{array}{c}K_{1}^{1} \\ \vdots \\ K_{100}^{1}\end{array}\right), K^{2}=\left(\begin{array}{c}K_{1}^{2} \\ K_{2}^{2} \\ K_{3}^{2}\end{array}\right)$ and $K^{3}=\left(\begin{array}{c}K_{1}^{3} \\ \vdots \\ K_{99}^{3}\end{array}\right)$ are such that the square matrices:

$\bar{A}^{1}=\left(\begin{array}{cccccc}K_{1}^{1} & 1 & 0 & \ldots & \ldots & 0 \\ K_{2}^{1} & 0 & \ddots & \ddots & & \vdots \\ \vdots & \vdots & \ddots & 1 & \ddots & \vdots \\ \vdots & \vdots & & \ddots & \ddots & 0 \\ K_{99}^{1} & 0 & \ldots & \ldots & 0 & 1 \\ K_{100}^{1} & 0 & \ldots & \ldots & \ldots & 0\end{array}\right), \bar{A}^{2}=\left(\begin{array}{ccc}K_{1}^{2} & 1 & 0 \\ & & \\ K_{2}^{2} & 0 & 1\end{array}\right)$ and $\bar{A}^{3}=\left(\begin{array}{cccccc}K_{1}^{3} & 1 & 0 & \ldots & \ldots & 0 \\ K_{2}^{3} & 0 & \ddots & \ddots & & \\ \vdots & \vdots & \ddots & 1 & \ddots & \vdots \\ \vdots & \vdots & & \ddots & \ddots & 0 \\ K_{39}^{3} & 0 & \ldots & \ldots & 0 & 1 \\ K_{100}^{3} & 0 & \ldots & \ldots & \ldots & 0\end{array}\right)$ are stable (it means that the real part of the eigenvalues of the $\bar{A}^{i}$ 's are negative).

$\Theta_{i}$ are given by: $\Theta_{1}=\left(\begin{array}{ccccc}\Omega & 0 & \ldots & \ldots & 0 \\ 0 & \Omega^{2} & \ddots & & \vdots \\ \vdots & \ddots & \ddots & \ddots & \vdots \\ \vdots & & \ddots & \ddots & 0 \\ 0 & \ldots & \ldots & 0 & \Omega^{100}\end{array}\right), \Theta_{2}=\left(\begin{array}{ccc}\Omega & 0 & 0 \\ 0 & \Omega^{2} & 0 \\ 0 & 0 & \Omega^{3}\end{array}\right)$ and 
$\Theta_{3}=\left(\begin{array}{ccccc}\Omega & 0 & \cdots & \cdots & 0 \\ 0 & \Omega^{2} & \ddots & & \vdots \\ \vdots & \ddots & \ddots & \ddots & \vdots \\ \vdots & & \ddots & \ddots & 0 \\ 0 & \ldots & \cdots & 0 & \Omega^{99}\end{array}\right)$ where $\Omega>0$ is the parameter of calibration of the observer, in our case $\Omega=3$.

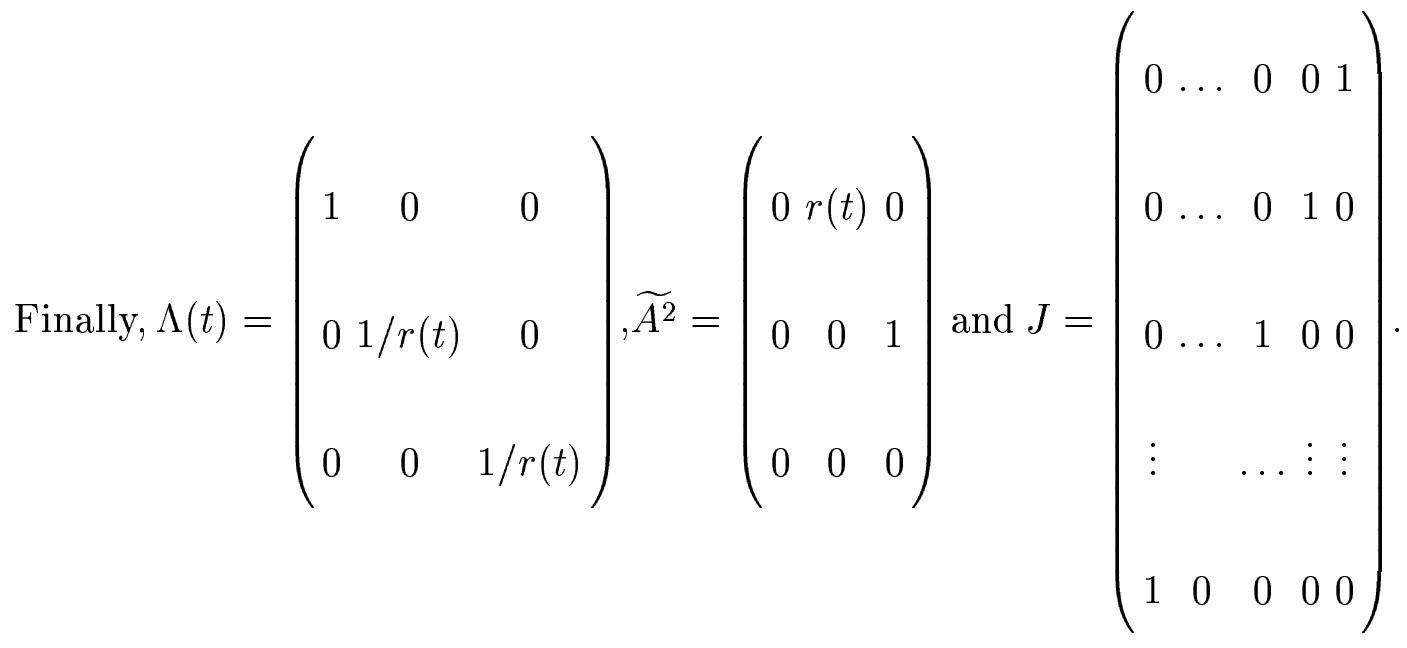


$a_{c}=$ Specific solid-fluid surface area $\left[\mathrm{m}^{-1}\right]$

$c_{p s}=$ Solid heat capacity $\left[J . k g^{-1} \cdot K^{-1}\right]$

$c_{p m g}=$ Fluid heat capacity $\left[J . k g^{-1} \cdot K^{-1}\right]$

$H=$ Total length of monolith $[\mathrm{m}]$

$h=$ Solid-fluid heat transfer coefficient $\left[W \cdot m^{-2} \cdot K^{-1}\right]$

$k_{D}=$ Solid-fluid mass transfer coefficient $\left[m . s^{-1}\right]$

$M=$ VOC molecular weight $\left[\mathrm{kg}_{\mathrm{m}} \mathrm{mol}^{-1}\right]$

$N^{\prime}=$ Number of transfer units for heat loss [-]

$P=$ Peclet number for solid-fluid heat transfer [-]

$P_{a x}=$ Axial Peclet number for heat conduction [-]

$P_{\theta}=\mathrm{P}$ corrected for the finite frequency [-]

$Q_{j}=$ External power supply $[W]$

$S=$ Total cross-section of the monolith $\left[\mathrm{m}^{2}\right]$

$T_{g 1}, T_{g 2}=$ Gas temperature in the upstream, downstream monolith $[\mathrm{K}]$

$T_{0}=$ Inlet and external temperature $[\mathrm{K}]$

$T_{\text {max }}=$ Maximum solid temperature in the RFR $[\mathrm{K}]$

$T_{s}$ or $T=$ Solid temperature $[\mathrm{K}]$

$t=$ Time $[\mathrm{s}]$

$u_{v 0}=$ Superficial gas velocity in the reference state $\left[m . s^{-1}\right]$

$x=$ Reduced abscissa, $2 z / H[-]$

$z=\operatorname{Abscissa}[\mathrm{m}]$

$\alpha=$ fraction of feed flow rate [-]

$\Delta H=$ Reaction enthalpy $\left[\right.$ J.mol $\left.^{-1}\right]$

$\Delta T_{a d}=$ Adiabatic temperature rise $[\mathrm{K}]$ 
$\varepsilon=$ fraction of open frontal area [-]

$\varphi(x)=$ Characteristic function of the catalytic monolith [-]

$\psi(x)=\varphi(x)\left(\omega_{1}+\omega_{2}\right) /\left(2 \omega_{10}\right)[-]$

$\rho=$ Fluid density $\left[k g \cdot m^{-3}\right]$

$\rho_{0}=$ Gas density in the reference state $\left[\mathrm{kg} \cdot \mathrm{m}^{-3}\right]$

$\rho_{s}=$ Solid density $\left[k g . m^{-3}\right]$

$\tau=$ Heat storage time constant $[\mathrm{s}]$

$\theta=$ Period of flow reversal $[\mathrm{s}]$

$\omega_{1 s u}, \omega_{2 s u}=$ VOC mass fraction of solid phase in the upstream,downstream

monoliths [-]

$\omega_{1}, \omega_{2}=$ VOC mass fraction in the upstream, downstream monoliths [-]

$\omega_{10}=$ VOC mass fraction in the feed [-]

$\xi=$ Reduced abscissa of the boundary between the inert and catalytic mono-

liths

$\xi_{\theta}=\xi$ corrected for the finite frequency [-]

$X=$ State vector

$X_{e}=$ Estimated state vector

$y=$ Filtered temperature measurements $[\mathrm{K}]$

$\Omega=$ Calibration parameter of the observer [-] 